psychiatric/behavioral services, onsite pharmaceutical, and durable medical equipment distribution, epidemiologic surveillance, and select laboratory services.

Aim: To describe how onsite medical care in the adapted environment of a large population shelter can provide comparable services and limit the direct impact on the local medical community.

Methods: A retrospective chart review of medical records was generated for all clinical encounters at the MMC. Data were sorted by daily census, disease surveillance, medical decision making, treatment, and transport destinations.

Results: $40.7 \%$ of registered evacuees utilized the MMC accounting for a total of 2,654 clinic visits by 1,560 unique patients representing all age groups. During the sustained MMC operations, $8 \%$ of patients required emergency transport and 500 additional patient transports were arranged for clinic appointments. No deaths occurred and no iatrogenic morbidity was reported.

Discussion: Medical care was provided for a large number of evacuees which mitigated the potential impact on the local medical infrastructure. The provision of medical services in a large population shelter may necessitate adaptation to the standard of care. However, despite the nontraditional clinical setting, care delivery was not compromised.

Prehosp. Disaster Med. 2019;34(Suppl. 1):s44-s45

doi:10.1017/S1049023X19001080

Typhoon Mangkhut Case Study: Household and Community Typhoon Preparedness in Hong Kong, a Densely Populated Urban City

Ms. Asta YT Man ${ }^{1,2}$, Prof. Emily Ying Yang Chan ${ }^{1,2}$,

Dr. Holly CY Lam ${ }^{1,2}$

1. Collaborating Centre for Oxford University and CUHK for Disaster and Medical Humanitarian Response (CCOUC), JC School of Public Health and Primary Care, The Chinese University of Hong Kong, Hong Kong, China

2. Division of Global Health and Humanitarian Medicine, JC School of Public Health and Primary Care, The Chinese University of Hong Kong, Hong Kong, China

Introduction: As a subtropical urbanized city in Southeast Asia, Hong Kong is prone to frequent typhoons. With an increasing number of severe typhoons, usual preparedness measures should be explored to assess their adequacy to safeguard health and wellbeing. Typhoon Mangkhut (2018) serves as an example of the successes and limitations of community preparedness for a severe typhoon.

Aim: To explore how Hong Kong residents prepared for Typhoon Mangkhut and whether their usual preparedness measures provided enough protection.

Methods: A population-based randomized telephone survey of Hong Kong residents $(n=521)$ was conducted soon after Typhoon Mangkhut's landing. Only residents aged 18 or above and understood Cantonese were included. Socio-demographic factors, types of typhoon and general preparedness, risk perception, and impacts from the typhoon were asked. Descriptive characteristics and univariate analysis were used to describe the patterns and associations.

Results: $8.6 \%$ of respondents felt their home was at high risk of danger during typhoons although $33.4 \%$ reported some form of impact from Mangkhut. Over 70\% reported doing at least one typhoon specific preparedness measure. Among those who practiced at least one typhoon specific preparedness measure, $37.2 \%(\mathrm{p}=0.002)$ were affected by the typhoon.

Discussion: Despite the high adaptation of preparedness measures, warranted by the frequent typhoons, Hong Kong residents were not adequately prepared for a severe typhoon. While the early warning system and evacuation of flood-prone areas mitigated some of the impact, unexpected effects such as flying air conditioners, roadblocks affecting employment, swaying buildings, and loss of power supply were not accounted for. Future preparedness for natural disasters which will become more extreme due to climate change and needs to account for unforeseen risks.

Prehosp. Disaster Med. 2019;34(Suppl. 1):s45

doi:10.1017/S1049023X19001092

\section{Typhoon Preparedness Measures of the Hong Kong Public} for Typhoon Mangkhut

Miss. Tiffany Yeung, Ms. Eva CC Lam

Hong Kong Jockey Club Disaster Preparedness and Response

Institute, Hong Kong

Introduction: Climate change has brought more extreme weather events to Hong Kong. The increasing number of powerful tropical cyclones that hammered Hong Kong in recent years reminded the territory to review typhoon preparedness of the community.

Aim: Typhoon Mangkhut slammed Hong Kong in September 2018 and caused significant devastation. Three weeks after the devastation, the Hong Kong Jockey Club Disaster Preparedness and Response Institute commissioned the Public Opinion Programme at The University of Hong Kong to conduct a survey to understand the general Hong Kong public's typhoon preparedness measures and their information seeking behaviors. Methods: A cross-sectional population-based anonymous telephone survey was conducted on a random sample of 1,018 Cantonese-speaking adults aged 18 or above.

Results: The most common typhoon preparedness measures were taping windows (45\%), followed by food stockpiling (18\%), and closing of doors/windows (10\%). Only $2 \%$ and $1 \%$ of the respondents were prepared for water and power outage, respectively. $36 \%$ of the respondents did not take any precautionary measures. $29 \%$ sought typhoon precautionary measures information from the mass media and 31\% of respondents relied on their previous experience. Other sources of information included government sources (7\%) and social media (7\%).

Discussion: Though no death cases were reported related to Typhoon Mangkhut, the effect of the superstorm caused over 300 casualties, blocked roads, and transportation chaos caused 